\title{
Successful Endoscopic Ultrasound Guided Drainage of Large Lesser Sac Abscess Cavity Postgastric Ulcer Sealed-Off Perforation: Novel Approach
}

\author{
Keyur Bhatt ${ }^{1} \quad$ Dhaval Mangukiya ${ }^{1} \quad$ Pankaj Desai ${ }^{1}$ \\ ${ }^{1}$ Department of GI Minimal Access Surgery, SIDS Hospital \& \\ Research Centre, Surat, Gujarat, India \\ 2Department of Clinical Research, SIDS Hospital \& Research Centre, \\ Surat, Gujarat, India
}

\author{
Krishna Parekh²
}

J Digest Endosc 2020;10:230-233

\begin{abstract}
Address for correspondence Keyur Bhatt, MS, MRCSEd, Department of GI Minimal Access Surgery, SIDS Hospital \& Research Centre, Majura Gate, Ring Road, Surat, Gujarat 395002, India (e-mail: drkeyurbhatt@gmail.com).
\end{abstract}

\author{
Abstract \\ Keywords \\ - endoscopic ultra- \\ sonography guided \\ drainage \\ - intraperitoneal \\ abscess cavity \\ - perforated peptic \\ ulcer
}

\begin{abstract}
Endoscopic ultrasonography (EUS) guided drainage is a beneficial and safe therapeutic tool that is used for the treatment of pancreatic mass and pseudocysts. Currently, the application of EUS-guided drainage is increasing for the treatment of different complications such as intraperitoneal abscess drainage following perforated peptic ulcer (PPU) or pararectal abscess. The intraperitoneal abscess with PPU is traditionally treated with surgical treatment. Herein, we report a lesser invasive drainage technique in the form of EUS-guided drainage for the treatment of intraperitoneal abscess.
\end{abstract}

\section{Introduction}

Perforated peptic ulcer (PPU) is an acute abdominal condition that usually gets complicated by intraperitoneal abscess. Such a situation usually requires a surgical treatment. ${ }^{1}$ Recently reported literature suggests that an application of endoscopic ultrasonography (EUS) guided drainage is feasible for the treatment of intraperitoneal abscess adjacent to the digestive tract. ${ }^{2,3}$ EUS-guided drainage is a well-established treatment of pancreatic pseudocysts. ${ }^{4}$ Here, we report a case in which EUS-guided drainage was successfully implemented to drain an intraperitoneal abscess following sealed-off peptic perforation.

\section{Case Report}

A 74-year-old female presented with a 7-day history of burning epigastric pain, low-grade fever, generalized myalgia, and anorexia. She was on nonsteroidal anti-inflammatory drugs for osteoarthritis, which she was taking on her own. Abdominal ultrasonography showed coagulated fluid collection, with air near the posterior part of the stomach body. A plain computed tomography (CT) scan of the abdomen revealed multiple communicating pockets of collection and air in the lesser sac. The patient was hospitalized for further work-up and treatment. Her laboratory examination revealed leukocyte count of $18,400 / \mathrm{mm}^{3}$, platelet count of $355,000 / \mathrm{mm}^{3}$, total protein level of $5.8 \mathrm{mg} / \mathrm{dL}$, serum albumin level of $3.4 \mathrm{mg} / \mathrm{dl}$, prothrombin time of 12.9 seconds, and international normalized ratio of 1.14. The patient underwent diagnostic upper gastrointestinal (GI) endoscopy and EUS, which revealed lesser sac abscess (-Fig. 1a) and multiple deep peptic ulcers of the greater and lesser curvature in the stomach (-Fig. 1b), with one large healing ulcer in the duodenum (-Fig. 1c). Clinical diagnosis of sealed-off peptic perforation was made in view of the current findings. There was no active perforation of any ulcer; hence, endoscopic ultrasound was used to locate and drain the abscess. EUS-guided drainage was performed under sedation using intravenous midazolam and fentanyl. The large lesser sac abscess cavity was visualized using a therapeutic endoscope and punctured by a 19-gauge needle. A guidewire was introduced through the needle and coiled within the large lesser sac abscess (-Fig. 2a). The punctured tract was dilated with a 6-Fr cystotome catheter using the Hurricane balloon to allow stent placement. A 20 -mm-long "Nagi" stent was inserted under combined endoscopic and fluoroscopic 

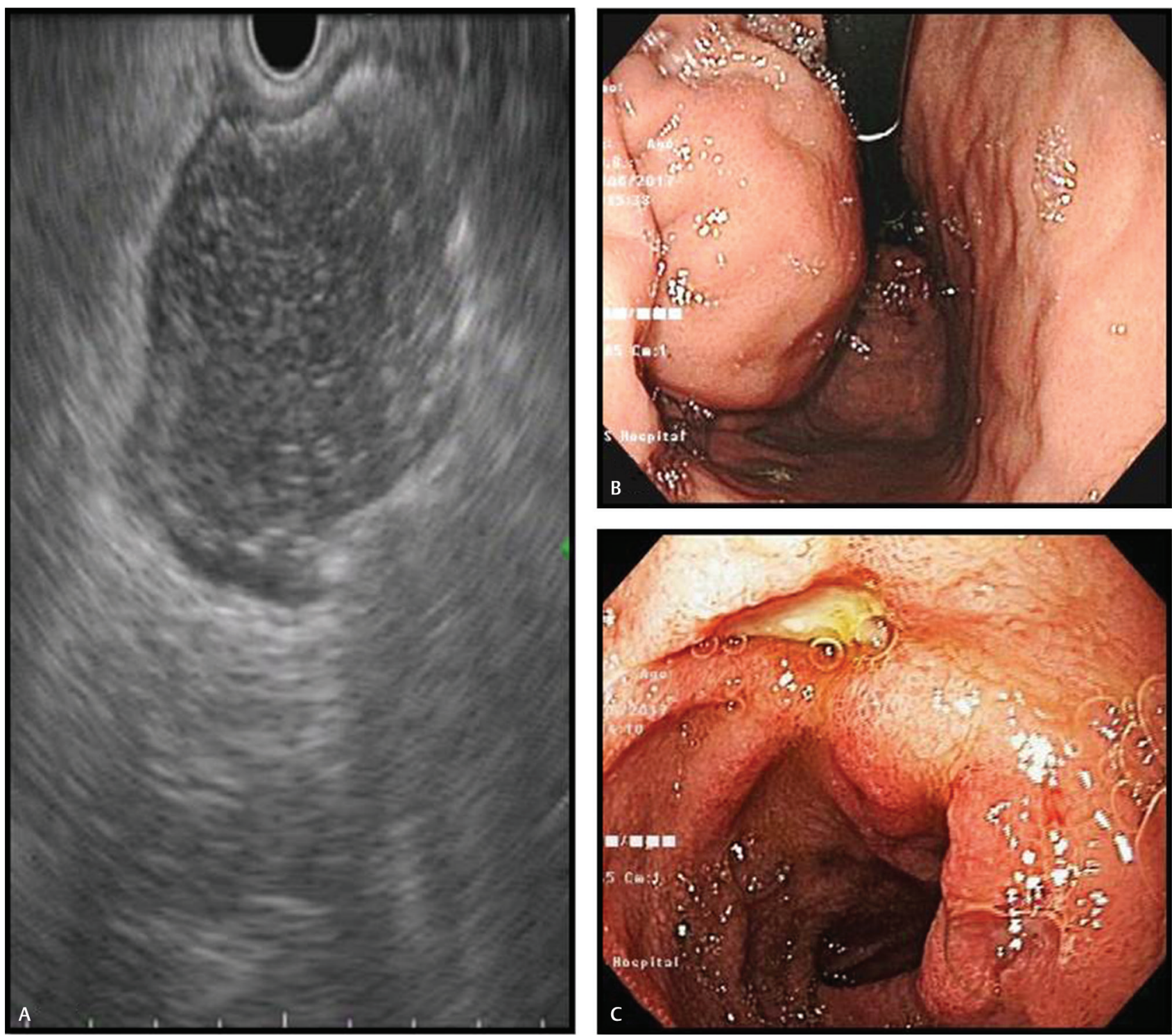

Fig. 1 Endoscopic and endoscopic ultrasonography examination of ulcer and abscess on the day of presentation. (a) Endoscopic ultrasonography showing lesser sac abscess. (b) Endoscopy showing sealed-off peptic perforation. (c) Duodenal ulcers.

guidance ( - Fig. 2 b). A short-term nasocystic drainage catheter was used for lavage. At the end of the procedure, a 7-Fr double-pigtail (DTP) stent was also kept through "Nagi" stent (-Fig. 2c). The pus was sent for culture and sensitivity. Klebsiella pneumoniae and Streptococcus gallolyticus species were detected in the culture report. She received intravenous antibiotic therapy of cefoperazone and sulbactam (3,000 mg/day) and proton pump inhibitors before and after drainage. On the third day of the procedure, abdominal ultrasonography was performed, which revealed a $23-\mathrm{mL}$ residual collection in relation to fundus and 2-mL collection in the posterior aspect of the stomach. EUS-guided single-shot drainage was performed for retrofundic collection; the "Nagi" stent was removed, and a DPT stent was kept in situ through the same opening. On the seventh day of admission, the patient was discharged after removal of the DPT stent and resumed oral diet. The patient was on clinical follow-up thereafter and oral proton pump inhibitors. On the 45th day of follow-up, GI endoscopy with
EUS was performed, which revealed no evidence of any ulcer or collection (-Fig. 3 ).

\section{Discussion}

The therapeutic value of EUS-guided drainage was first recognized in 1992 for the treatment of pancreatic pseudocyst. ${ }^{4}$ Currently, EUS-guided drainage usage for an intraperitoneal abscess resulting from PPU is still in inception. The literature search revealed only one published case report, which revealed EUS-guided fine-needle aspiration for the treatment of complication of PPU. ${ }^{3}$ This case report demonstrates our experience of treating intraperitoneal abscess resulting from PPU with EUS-guided drainage.

Usually, an intraperitoneal abscess formed after PPU is treated by surgery/laparoscopy with lavage and drainage along with suture repair of perforation. ${ }^{1}$ The surgical treatment does carry morbidity. ${ }^{1,3}$ In this case, the collection 

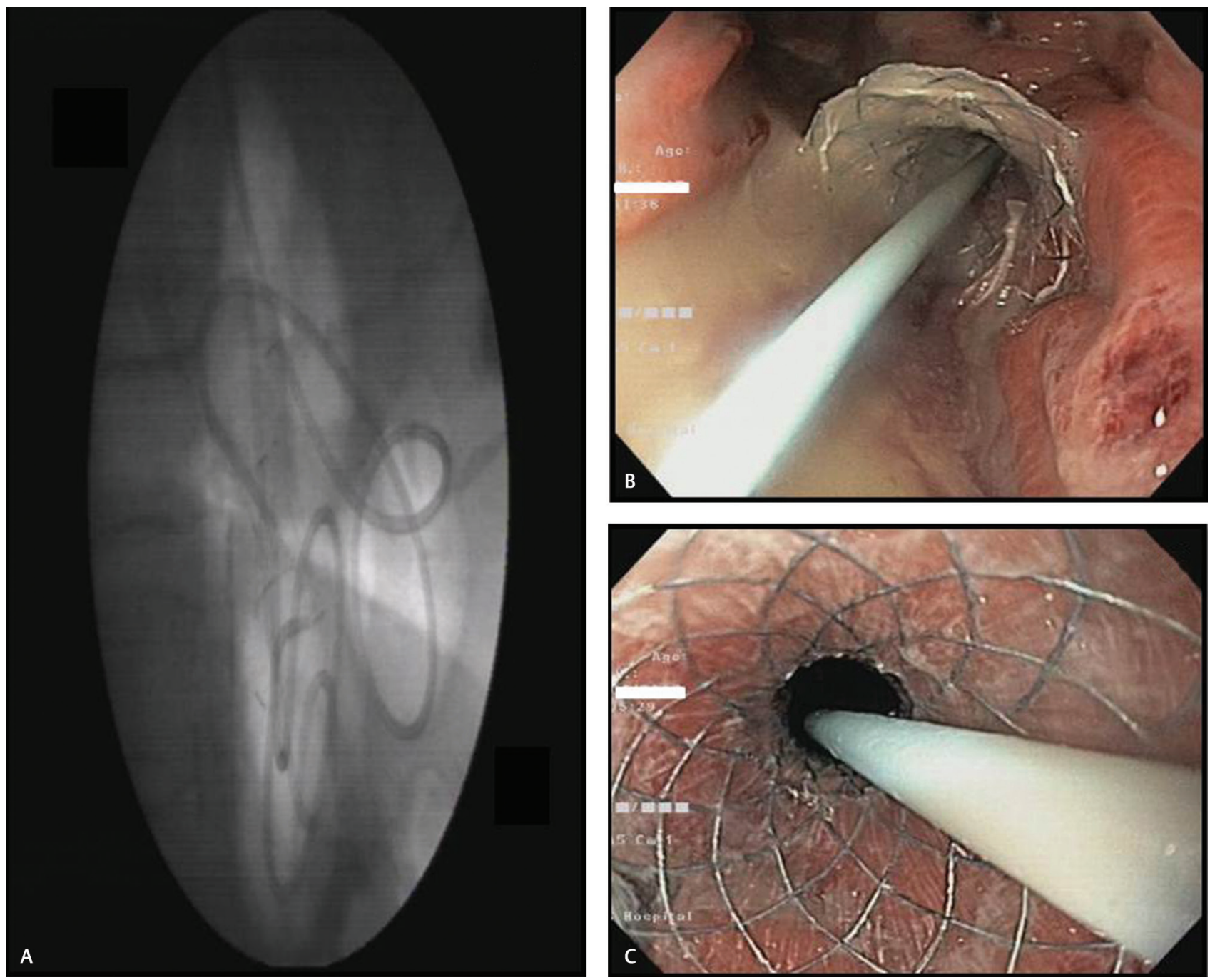

Fig. 2 Endoscopic ultrasonography-guided drainage technique. (a) Guidewire placement under endoscopic ultrasonography guidance. (b) Placement of "Nagi" stent for the drainage of pus. (c) Double-pigtail stent through Nagi stent.

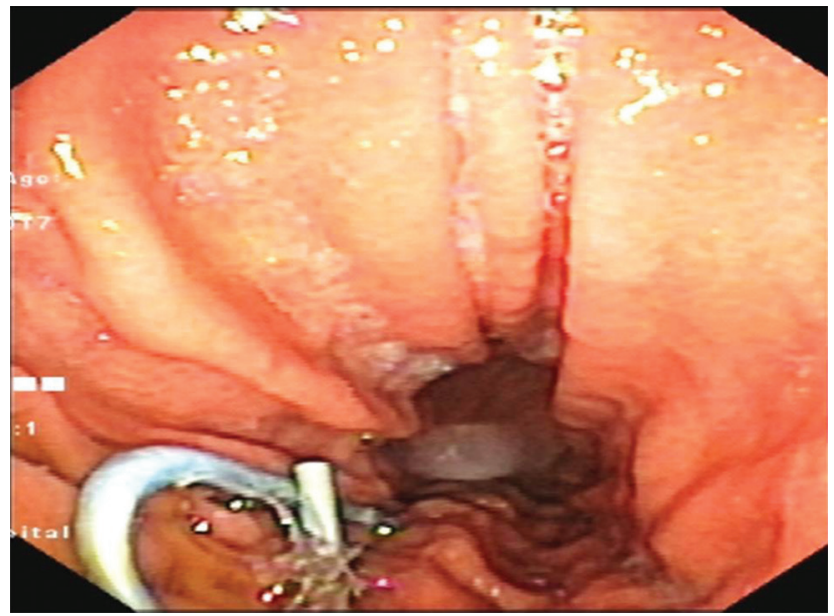

Fig. 3 Endoscopic examination on the 45th day, showing no evidence of any ulcer or perforation.

was situated only in lesser sac without contaminating the greater sac. Hence, therapeutic treatment with the help of EUS was decided as a modality. The abscess was then treated successfully with EUS-guided drainage. In addition, a complete general anesthesia was also avoided in this patient. Surgical complications can be minimized when elderly patients are treated with the lesser invasive method of EUS-guided drainage whenever feasible. The procedure's safety and efficacy need to be confirmed by larger clinical trials.

\section{Conclusion}

EUS-guided drainage is a lesser invasive and useful technique in the treatment of intraperitoneal (lesser sac) abscess cavity formed after PPU. In future, the indications for EUS-guided drainage will be established by the accumulation of further clinical experience.

\section{Author's Contribution}

K. B. and P. D. made diagnosis of large lesser sac abscess cavity postgastric ulcer sealed-off perforation. K. B. and D. M. conceived the present idea of implementation of a lesser invasive technique of EUS-guided drainage. D. M. encouraged K. B. and P. D. K. P. wrote the manuscript in consultation with K. B. 


\section{Funding}

Nil.

\section{Conflicts of Interest}

There are no conflicts of interest.

\section{References}

1 Søreide K, Thorsen K, Harrison EM, et al. Perforated peptic ulcer. Lancet 2015;386(10000):1288-1298

2 Mandai K, Shirakawa A, Uno K, Yasuda K. Endoscopic ultrasound-guided drainage of intra-abdominal abscess after gastric perforation in a patient receiving ramucirumab and paclitaxel for advanced gastric cancer. Case Rep Oncol 2017; 10(1):15-20

3 Hara A, Fukunishi S, Sasaki Y, Sanomura M, Higuchi K. EUS-FNA can be an effective procedure for the transesophageal drainage of a patient with intra-peritoneal abscess. Clin J Gastroenterol 2015;8(4):212-216

4 Sadik R, Kalaitzakis E, Thune A, Hansen J, Jönson C. EUSguided drainage is more successful in pancreatic pseudocysts compared with abscesses. World J Gastroenterol 2011;17(4):499-505 